\title{
Profile and health-related quality of life of Ghanaian stroke survivors
}

\author{
This article was published in the following Dove Press journal: \\ Clinical Interventions in Aging \\ 8 October 2014 \\ Number of times this article has been viewed
}

\author{
Eric S Donkor ${ }^{1,2}$ \\ Mayowa O Owolabi ${ }^{3}$ \\ Patrick O Bampoh ${ }^{4}$ \\ Philip K Amoo 5 \\ Thor Aspelund ${ }^{2,6}$ \\ Vilmundur Gudnason ${ }^{2,6}$ \\ 'College of Health Sciences, \\ University of Ghana, Accra, Ghana; \\ ${ }^{2}$ Centre for Public Health Sciences, \\ University of Iceland, Reykjavík, \\ Iceland; ${ }^{3}$ Department of Medicine, \\ University of Ibadan and University \\ College Hospital, Ibadan, Nigeria; \\ ${ }^{4}$ Tamale Central Hospital, Tamale, \\ Ghana; ${ }^{5}$ Public Health Unit, Korle-Bu \\ Teaching Hospital, Accra, Ghana; \\ ${ }^{6}$ Icelandic Heart Association Research \\ Institute, Kopavogur, Iceland
}

Background: Stroke is a leading cause of mortality with a major effect on health-related quality of life (HRQoL). There are no previous studies exploring HRQoL among stroke survivors in Ghana, despite the increasing public health significance of the disease in this country. Here we describe the profile of and factors associated with HRQoL among stroke survivors in Ghana. Methods: This was a cross-sectional study involving 156 stroke survivors and 156 age- and sex-matched, apparently healthy controls. A robust HRQoL questionnaire involving seven domains was used to collect data from the study participants. Clinical epidemiology data were also collected from stroke survivors on parameters such as stroke severity and risk factors. Statistical analyses were performed on the interrelationships among the study variables.

Results: The mean ages of the stroke survivors and healthy controls were 58.0 (standard deviation, 11.4) and 57.6 (standard deviation, 12.0) years, respectively. Fifty-three percent (86) of the stroke survivors had mild stroke and 35.3\% (55) had moderate stroke, whereas $12.2 \%$ (19) had severe stroke. Ischemic infarction was the prevalent stroke subtype (78.1\%). Hypertension was the most common risk factor (89\%) among the stroke survivors, followed by diabetes (29\%). HRQoL scores ranged from $57.7 \%$ (cognitive domain) to $80.0 \%$ (spirit domain) for stroke survivors, whereas HRQoL scores of the control group ranged from $65.6 \%$ (cognitive domain) to $85.2 \%$ (soul domain). For each HRQoL domain, significantly higher scores were observed for the control group compared with the stroke survivors, at $P<0.05$. Determinants of HRQoL of stroke survivors in multivariate analysis included age, stroke severity, poststroke duration, stroke recurrence, frequency of laughter, and negative emotions.

Conclusion: The most affected HRQoL domains are of the physical, psychoemotional, and cognitive domains. Rehabilitation of stroke patients in this region should include interventions targeted at these domains and modifying the statistical determinants of HRQoL where possible.

Keywords: quality of life, stroke, domain, Ghana

\section{Introduction}

The concept of health-related quality of life (HRQoL) is highly important in the assessment of the multifaceted effect of disease on the patient's life and evaluation of the utility and disability associated with various health states. ${ }^{1,2}$ HRQoL measures encompass emotional, physical, social, and subjective feelings of well-being and hence, can be used in identifying and prioritizing areas of need of individual patients and patients with special needs. ${ }^{3,4} \mathrm{HRQoL}$ measures are also useful in the evaluation of the effectiveness and cost benefit of various old and emerging prophylactic, therapeutic, and rehabilitative interventions. ${ }^{1-4}$ These instruments facilitate patientcaregiver communication and clinical decision making and uncover hidden problems. In general, few stroke studies have applied HRQoL, and these studies were mainly carried out in the developed world.
Correspondence: Eric S Donkor University of Ghana, Medical School, College of Health Sciences,

Accra, Ghana

Email esampane-donkor@chs.ug.edu.gh 
At this time, the majority of stroke morbidity and mortality cases occur in sub-Saharan Africa, a situation that has been attributed to the rapid epidemiological transition occurring in this region, increasing the risk factors for vascular disease. ${ }^{5,6}$ In Ghana, stroke ranks among the top three causes of mortality and is probably the most important cause of disability. ${ }^{7}$ The few stroke studies in Ghana have focused on stroke risk factors and the burden of the disease, ${ }^{8-11}$ and as such, no study has investigated the quality of life of stroke survivors in the country. Because of the increasing number of stroke survivors and the limited rehabilitation facilities in Ghana, it is essential to identify and modulate the factors affecting HRQoL to promote maximal HRQoL improvements in these patients. Recently, evidence-based medicine has become a key priority of the Ghanaian health system, and it is imperative that studies aimed at understanding the nature of stroke in the Ghanaian population, such as quality of life, contribute to management of the disease.

The objectives of the study were to describe the profile of stroke survivors in Ghana and to evaluate their HRQoL and the associated determinants.

\section{Methods}

\section{Study area and sampling}

The study was carried out from January 2012 to September 2013 at the physiotherapy unit of two major hospitals in Ghana; namely, Korle-Bu Teaching Hospital and Tema General Hospital. The two healthcare facilities were selected for the study on the basis of their high stroke patient turnout. Korle-Bu Teaching Hospital is located in Accra, the capital city of Ghana, whereas Tema General Hospital is located in Tema, which is Ghana's most industrialized city. Both healthcare facilities are state-owned and operate the national health insurance scheme introduced in Ghana in 2004.

This was a questionnaire-based, cross-sectional study comprising stroke patients and healthy controls. Using a 95\% confidence level, 11\% estimated stroke prevalence reported previously, ${ }^{9}$ and 5\% allowable error, 78 consecutive stroke patients who developed stroke at least 1 month before the time of interview were enrolled at each of the physiotherapy units. The absence of a cutoff point for maximum duration was to permit the determination of the influence of duration after stroke on HRQoL, whereas the 1-month minimum duration was chosen to exclude acute cases of stroke that were yet to stabilize. The recruitment of stroke patients was based on definite clinical and/or radiological diagnosis of stroke, and patients with communication problems who had reliable proxies were also included in the study. Patients with ambiguous diagnosis of stroke were excluded from the study. A control group comprising 78 age- and sex-matched subjects without clinical evidence of stroke or any history of the disease was recruited from the environs of each of the healthcare facilities. The main purpose of the control group was for comparison of HRQoL between stroke survivors and healthy individuals.

\section{The study instrument}

The questionnaire used in this study was comprised of four main sections. The first section was about respondents' demographic details, including age, sex, marital status, religion, education, and income. The second section of the questionnaire was on clinical and epidemiological information related to stroke and was administered only to the stroke patients. This aspect of the questionnaire contained information on duration, side, subtype, severity, and number of strokes. It also had information on stroke risk factors and neurological impairments. Data related to this part of the questionnaire were obtained through interviews and information from folders of the patients. Information on stroke subtype was based solely on radiological diagnosis (computed tomography scan). The third section of the study questionnaire was on HRQoL of stroke and was administered to both stroke patients and the healthy control group. The HRQoL aspect of the questionnaire involved two dimensions and seven domains. The physical dimension included physical, psychoemotional, cognitive, and ecosocial domains, whereas the spiritual dimension included soul, spirit, and spiritual interaction domains. ${ }^{12}$ Unlike other HRQoL instruments, the one used in this study (Health Related Quality of Life in Stroke Patients [HRQoLSIP-40]) had been previously validated in similar studies in West Africa and was found to demonstrate good content, construct, and discriminant validity and internal consistency reliability. ${ }^{2,12}$ The fourth section of the questionnaire evaluated knowledge of stroke risk factors among the stroke patients and healthy controls, and the significance of this was to evaluate the possible association between stroke and awareness of its risk factors.

\section{Ethics statement}

The study was approved by the Ghana Health Service Ethics Review Committee (number GHS-ERC: 03/07/11), and informed consent was obtained from the study participants.

\section{Data analysis}

Data were analyzed using SPSS version 11.0 (SPSS Inc., Chicago, IL, USA). Demographic variables in both study 
populations were summarized and compared using the Student's $t$-test for numeric variables and chi-square tests for categorical variables. Stroke risk factors, subtypes, and other clinical parameters of stroke patients were described by prevalence rates and frequencies. Awareness of stroke risk factors was compared between stroke patients and the control group by chi-square.

HRQoL data were analyzed using gold standard methods widely reported in HRQoL literature. The HRQoL scores for each domain were computed by the Likert method, with a high score indicating better quality of life. ${ }^{1-3}$ The Likert scale range was $1-5$, and domain scores were transformed into a scale of $0-100$, with 0 indicating worst health and 100 best health. The overall HRQoL score was computed by finding the arithmetic mean of the various domain scores. Stroke severity was measured by the stroke levity scale, which was computed based on the following formula: stroke levity = maximum power in the dominant hand + maximum power in the weaker lower limb + mobility score -1 (if aphasia present). ${ }^{13}$ The scores were then stratified as severe stroke, moderate stroke, and mild stroke.

Initially, univariate associations were performed between each HRQoL domain and all demographic, clinical, and psychoemotional features: analysis of variance/Student's $t$-test was used for numeric variables, whereas chi-square test was used for categorical variables. Subsequently, variables significantly associated with the HRQoL domains were used in multiple regression analysis to identify determinants of HRQoL.

\section{Results \\ Demographic features of the study participants}

The demographic features of the study participants are reported in Table 1. The gender distribution of the stroke survivors and healthy controls were the same, with 97 men and 59 women. The age distributions of the two groups of study subjects were also very similar: mean age of stroke survivors was 58.0 (standard deviation, 11.4) years, whereas that of healthy controls was 57.6 (standard deviation, 12.0) years. Although secondary education was the most common educational level among the stroke survivors (49.0\%), tertiary education was the most common among the healthy controls $(37.9 \%)$. Among both the stroke survivors and healthy controls, the majority were Christians, married, and had a monthly income range of 100-999 Ghanaian cedi (USD 50-500). Education, marital status, and income were significantly different between the stroke survivors and healthy controls.

\section{Clinical parameters and stroke risk factors}

One hundred and thirty-two of the stroke survivors (84.1\%) had experienced stroke once, 23 (14.7\%) had two stroke episodes, and one (1.2\%) had three stroke episodes; overall, the recurrence of stroke was $15.4 \%$. The period during which the stroke survivors had lived with the disease ranged from 3 months to 25 years, with a mean of 2 years and 5 months. In $51.3 \%$ (79) of the stroke cases, the disease had affected the right half of the body, whereas for $48.7 \%$ (75), the left half of the body was affected. Aphasia and sexual dysfunction were reported in $34.2 \%(51)$ and $57.7 \%$ (90) of the patients, respectively, whereas negative feelings such as fear and depression were present in $83.3 \%$ (130) cases. The drugs used by the stroke patients were mainly antihypertensives and pain killers, and none of the patients had thrombolytic therapy.

The stroke levity scale classified stroke severity as follows: severe stroke, $12.2 \%$ (19); moderate stroke, 35.3\% (55); and mild stroke, $52.6 \%$ (82). On the basis of radiological information (available for 32 patients), 18.8\% of the strokes were hemorrhagic, $78.1 \%$ were infarctions, and 3.1\% were both hemorrhagic and ischemic infarctions.

The distribution of stroke risk factors among the stroke survivors is reported in Figure 1. Overall, hypertension was the most common risk factor $(89 \%)$, followed by diabetes (29\%), alcohol consumption (28\%), and high cholesterol (25\%); the least prevalent risk factor was heart disease (7\%). Assessment of knowledge of stroke risk factors showed that $61 \%$ (95) of the stroke survivors could not correctly identify any stroke risk factor, $28 \%$ (44) correctly listed one stroke risk factor, and 11\% (17) correctly listed two or more risk factors. In the case of the control group, 47\% (73) could not correctly identify any stroke risk, 38\% (59) correctly listed one established stroke risk factor, and 15\% (24) correctly listed two or more risk factors. Knowledge of stroke risk factors was significantly better among the control group than the stroke survivors $(P=0.011)$.

\section{HRQoL}

Comparison of mean HRQoL scores between stroke survivors and healthy controls is reported in Table 2. Among stroke survivors, HRQoL scores ranged from $57.7 \%$ (cognitive domain) to $80.0 \%$ (spirit domain), whereas the HRQoL scores of the control group ranged from $65.6 \%$ (cognitive domain) to 
Table I Demographic features of the study participants

\begin{tabular}{|c|c|c|c|}
\hline Variable & Stroke group, n (\%) & Control group, n (\%) & Significance \\
\hline Sex & & & Identical \\
\hline Male & $97(62.2)$ & $97(62.2)$ & \\
\hline Female & $59(37.8)$ & $59(37.8)$ & \\
\hline Age range, years* & & & $P>0.05$ \\
\hline $20-29$ & $\mathrm{I}(0.6)$ & I $(0.6)$ & \\
\hline $30-39$ & $8(5.1)$ & $8(5.1)$ & \\
\hline $40-49$ & $25(16)$ & $25(16)$ & \\
\hline $50-59$ & $51(32.7)$ & $51(32.7)$ & \\
\hline $60-69$ & $45(28.8)$ & $45(28.8)$ & \\
\hline $70-79$ & $20(12.8)$ & $20(12.8)$ & \\
\hline 80-89 & $5(3.2)$ & $5(3.2)$ & \\
\hline Education & & & $P<0.05$ \\
\hline None & II (7.I) & $12(7.9)$ & \\
\hline Primary & $14(9.0)$ & $47(30.1)$ & \\
\hline Secondary & $76(48.7)$ & $39(25.0)$ & \\
\hline Tertiary & $55(35.3)$ & $58(37.2)$ & \\
\hline Marital status & & & $P<0.05$ \\
\hline Married & II2 (7I.8) & $108(69.2)$ & \\
\hline Single & $9(5.8)$ & $16(10.3)$ & \\
\hline Separated & $3(1.9)$ & $13(8.0)$ & \\
\hline Divorced & $8(5.1)$ & $4(2.6)$ & \\
\hline Widowed & $24(15.4)$ & $15(9.6)$ & \\
\hline Religion & & & $P>0.05$ \\
\hline Christian & 149 (95.5) & | 42 (9l.0) & \\
\hline Moslem & $6(3.8)$ & $14(9.0)$ & \\
\hline Traditional religion & I $(0.6)$ & $0(0)$ & \\
\hline Income, Ghananian cedi & & & $P<0.05$ \\
\hline$<100$ & $7(4.5)$ & $19(12.2)$ & \\
\hline $100-999$ & $86(55.1)$ & $85(54.5)$ & \\
\hline $1,000-1,999$ & $9(5.8)$ & $22(14.1)$ & \\
\hline $2,000-2,999$ & $4(2.6)$ & $7(4.5)$ & \\
\hline$\geq 3,000$ & I (0.6) & $3(1.9)$ & \\
\hline Unemployed & $49(31.4)$ & $21(13.5)$ & \\
\hline
\end{tabular}

Note: *Mean age of stroke survivors and healthy controls were 58.0 (standard deviation, 11.4) and 57.6 (standard deviation, 12.0) years, respectively.

$85.3 \%$ (soul domain). For each HRQoL domain, significantly higher scores were observed for the control group compared with the stroke survivors. The most affected HRQoL domains of the stroke survivors were the physical, psychoemotional, and cognitive domains. Comparison of the mean HRQoL scores between stroke survivors at Korle-Bu Teaching Hospital and Tema General Hospital showed there were no significant differences for the physical, soul, spirit, and spiritual interaction domains (Table 3). However, stroke survivors at Tema General Hospital had significantly higher HRQoL scores for the psychoemotional, cognitive, and ecosocial domains than stroke survivors at Korle-Bu Teaching Hospital (Table 3).

In the univariate analysis, HRQoL was significantly associated with several demographic features. Age was associated with the physical domain $(P=0.001)$, cognitive domain $(P=0.006)$, ecosocial domain $(P=0.001)$, and soul domain $(P=0.008)$. There was no significant difference between men and women for any of the HRQoL domains. Income level and religion were, respectively, associated with ecosocial domain ( $P=0.008)$ and psychoemotional domain $(P=0.026)$, whereas educational level was associated with both cognitive domain $(P=0.034)$ and ecosocial domain $(P=0.013)$.

In the univariate analysis, HRQoL was also significantly associated with several clinical/psychoemotional features. With the exception of cognitive domain and spirit interaction domain, stroke severity was associated with all the other HRQoL domains $(0.0001<P<0.043)$. Stroke frequency was associated with cognitive domain $(P=0.006)$, psychoemotional domain $(P=0.032)$, and ecosocial domain $(P=0.015)$, whereas stroke duration was associated with the spirit domain $(P=0.008)$. Stroke subtype and the side of the body affected by stroke, however, did not affect HRQoL. Among the various risk factors of stroke, only diabetes and heart disease affected HRQoL; diabetes was associated with physical domain $(P=0.033)$, and heart disease was associated with cognitive domain $(P=0.039)$ and ecosocial domain 


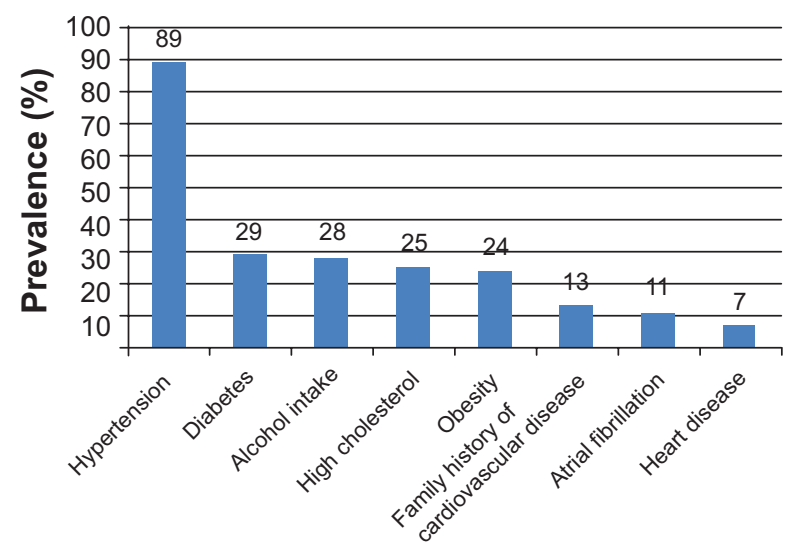

Figure I Distribution of stroke risk factors among Ghanaian stroke survivors.

$(P=0.044)$. Frequency of laughter was associated with all the HRQoL domains $(0.0001<P<0.024)$, whereas negative feelings were only associated with psychoemotional domain $(P<0.0001)$.

Variables included in the multivariate regression model were age, poststroke duration, type and number of strokes, stroke levity score, and Likert-scale responses to laughter frequency and negative feelings frequency. Independent determinants of HRQoL identified through the regression analysis are reported in Table 4. Younger age was associated with better physical and soul HRQoL domains. Shorter stroke duration was associated with better spirit HRQoL, whereas longer duration was associated with better psychoemotional HRQoL. Lower frequency of stroke determined better cognitive HRQoL. With the exception of spiritual interaction domain, stroke severity (levity) was associated with all the HRQoL domains, with mild stroke determining better quality of life. Frequent negative feelings or emotions were associated with poorer psychoemotional HRQoL. With the exception of physical and spiritual domains, frequency of laughter was associated with all the HRQoL domains, with higher laughter frequency determining better quality of life.

\section{Discussion}

In this study, we investigated the profile and HRQoL of stroke survivors in Ghana. The mean age of stroke survivors in this study ( 58 years) is similar to that reported by a Nigerian study in which the mean age of stroke survivors was 59 years. ${ }^{14}$ In comparison, the mean age of stroke survivors in a German population was much greater, at 69 years. ${ }^{14}$ This disparity may be a result of the relatively longer life expectancy in the Western world and the fact that age-specific stroke incidence is relatively higher in younger age groups in sub-Saharan Africa. The male-to-female ratio of the stroke survivors was 1.6:1, which concurs with both a postmortem stroke study and an inpatient stroke study carried out in Ghana. ${ }^{9,11}$ In contrast, female predominance has been reported in stroke survivors in Chicago, IL, USA; Poland; and Melbourne, Australia. ${ }^{15-17}$

The distribution of stroke subtypes in this study contrasts with distribution in previous studies in Ghana. Studies carried in 1954 and 1981 showed that hemorrhagic stroke was the predominant stroke subtype in Ghana and accounted for approximately $90 \%$ of stroke deaths. ${ }^{10,18}$ However, studies carried out in 1994 and 1998 showed that the proportion of hemorrhagic strokes had declined to $60 \%$, and cerebral infarction was becoming more common in the country. ${ }^{9}$ The current study shows that cerebral infarction may be the predominant stroke type in Ghana, accounting for about $78 \%$ of stroke cases. A similar evolution of the distribution of stroke subtypes has been previously reported for England and Wales. ${ }^{19}$ Despite the small size, our data on stroke subtype agree with an international stroke study of 3,000 cases in which the prevalence of hemorrhagic and ischemic infarctions were $22 \%$ and $78 \%$, respectively. ${ }^{20}$

Hypertension was the most important stroke risk factor in this study, an observation that has been reported by several other studies in different parts of the world. ${ }^{20-23}$ In Ghana, there is a high prevalence of hypertension in the general population, which may partly account for the trend of increasing stroke cases in the country. ${ }^{23,24}$ There is also

Table 2 HRQoL scores of Ghanaian stroke survivors and healthy controls

\begin{tabular}{llll}
\hline HRQoL domain & Stroke group, mean \pm SD & Control group, mean \pm SD & $P$-value \\
\hline Physical domain & $62.8 \pm 15.8$ & $83.1 \pm 12.7$ & $<0.000 I^{*}$ \\
Psychoemotional domain & $58.2 \pm 15.7$ & $78.4 \pm 12.2$ & $<0.000 I^{*}$ \\
Cognitive domain & $57.7 \pm 16.2$ & $65.6 \pm 10.6$ & $<0.000 I^{*}$ \\
Ecosocial domain & $72.1 \pm 14.3$ & $77.6 \pm 10.4$ & $0.000 I^{*}$ \\
Soul domain & $78.3 \pm 12.5$ & $85.3 \pm 9.7$ & $<0.000 I^{*}$ \\
Spirit domain & $80.0 \pm 13.6$ & $85.1 \pm 9.9$ & $0.0002^{*}$ \\
Spiritual interaction domain & $73.8 \pm 14.8$ & $81.2 \pm 11.0$ & $<0.000 I^{*}$ \\
HRQoL sum & $69 \pm 13.3$ & $79.5 \pm 10.9$ & $<0.000 I^{*}$ \\
\hline
\end{tabular}

Note: $* P$-value is significant.

Abbreviations: HRQoL, health-related quality-of-life; SD, standard deviation. 
Table 3 HRQoL scores of stroke survivors at Korle-Bu Teaching Hospital and Tema General Hospital

\begin{tabular}{llll}
\hline HRQoL domain & Korle-Bu Teaching Hospital, mean \pm SD & Tema General Hospital, mean \pm SD & $P$-value \\
\hline Physical domain & $60.4 \pm 15.5$ & $65.1 \pm 15.7$ & $0.065 I$ \\
Psychoemotional domain & $55.5 \pm 15.0$ & $60.9 \pm 16.2$ & $0.0325^{*}$ \\
Cognitive domain & $50.8 \pm 13.7$ & $64.6 \pm 15.7$ & $<0.000 I^{*}$ \\
Ecosocial domain & $65.8 \pm 11.6$ & $78.5 \pm 14.0$ & $0.000 I^{*}$ \\
Soul domain & $76.6 \pm 11.6$ & $80.0 \pm 13.3$ & 0.0915 \\
Spirit domain & $78.1 \pm 12.6$ & $81.9 \pm 14.4$ & 0.0780 \\
Spiritual interaction domain & $74.5 \pm 13.0$ & $73.0 \pm 16.3$ & 0.5343 \\
HRQoL sum & $70.0 \pm 13.3$ & $83.4 \pm 15.1$ & $<0.000 I^{*}$ \\
\hline
\end{tabular}

Note: $*$-value is significant.

Abbreviations: HRQoL, health-related quality-of-life; SD, standard deviation.

a high level of undiagnosed hypertension and poor compliance to antihypertensive drugs in the country, ${ }^{10}$ which are important issues, given the relationship between stroke and hypertension. Although prevalence of hypertension in this study $(82 \%)$ is similar to that reported in a Nigerian study (87\%), we observed significantly higher prevalence of other risk factors such as diabetes, smoking, alcohol consumption, and atrial fibrillation in the Ghanaian study. ${ }^{14}$ In comparison, prevalence rates of atrial fibrillation and ischemic heart disease among stroke patients $(20 \%-30 \%)$ in the United Kingdom and Germany are higher than what we observed among the Ghanaian stroke patients (7\%-11\%). ${ }^{14,22}$ This highlights the racial disparity of stroke risk factors, which is thought to be important in the geographical distribution of stroke subtypes. ${ }^{6,17}$ We could not investigate the relationship between stroke risk factors and stroke subtypes because of the limited data on stroke subtypes in our sample. However, evidence from others studies shows that hemorrhagic stroke is more associated with hypertension, ${ }^{25-27}$ whereas ischemic stroke is more associated with smoking and cardiac disease. ${ }^{26,28}$ Stroke recurrence in this study was $15.4 \%$, which is quite high and highlights the need for stroke secondary

Table 4 Determinants of HRQoL of Ghanaian stroke survivors identified through regression analysis

\begin{tabular}{|c|c|c|c|}
\hline HRQoL domain and determinant & $t$ & $P$ & $\beta$ \\
\hline \multicolumn{4}{|l|}{ Physical domain } \\
\hline Age & -2.454 & 0.016 & -0.212 \\
\hline Stroke levity & 5.307 & $<0.000$ I & 0.472 \\
\hline \multicolumn{4}{|l|}{ Psychoemotional domain } \\
\hline Stroke levity & 4.57 & $<0.0001$ & 0.378 \\
\hline Laughter frequency & 6.302 & $<0.0001$ & 0.495 \\
\hline Negative feelings & 3.973 & $<0.0001$ & 0.296 \\
\hline Stroke duration & 3.993 & 0.028 & 1.088 \\
\hline \multicolumn{4}{|l|}{ Cognitive domain } \\
\hline Stroke levity & 4.915 & $<0.0001$ & 0.445 \\
\hline Laughter frequency & 2.669 & 0.009 & 0.23 \\
\hline Stroke frequency or number & -2.246 & 0.027 & -0.208 \\
\hline \multicolumn{4}{|l|}{ Ecosocial domain } \\
\hline Age & -0.209 & 0.041 & 0.041 \\
\hline Stroke levity & 4.329 & $<0.000$ I & 0.388 \\
\hline Laughter frequency & 3.108 & 0.003 & 0.265 \\
\hline \multicolumn{4}{|l|}{ Soul domain } \\
\hline Age & -2.375 & 0.02 & -0.218 \\
\hline Stroke levity & 2.807 & 0.006 & 0.265 \\
\hline Laughter frequency & 3.334 & 0.001 & 0.3 \\
\hline \multicolumn{4}{|l|}{ Spirit domain } \\
\hline Stroke levity & 2.172 & 0.032 & 0.222 \\
\hline Stroke duration & -3.125 & 0.002 & -0.3 \\
\hline \multicolumn{4}{|l|}{ Spiritual interaction domain } \\
\hline Laughter frequency & 2.585 & 0.011 & 0.266 \\
\hline
\end{tabular}

Abbreviations: HRQoL, health-related quality-of-life; $\beta$, coefficient; $P$, probability value; $t, t$ statistic (coefficient divided by standard error). 
prevention efforts. Interestingly, knowledge of stroke risk factors was significantly better among the control group compared with the stroke patients, indicating a possible association between stroke and awareness of its risk factors. However, this requires further investigation, as several factors such as education and income were significantly different between the stroke patients and healthy controls.

To the best of our knowledge, this study is the first on HRQoL of stroke survivors in Ghana and one of the very few to report on the subject in sub-Saharan Africa. As reported by other studies, we observed multidomain impairment of the stroke patients compared with the control group, particularly for the physical, psychoemotional, and cognitive domains. ${ }^{2,17,29,30}$ It is difficult to explain the differences in HRQoL of stroke survivors between the two study hospitals. However, these differences probably indicate that HRQoL associated with stroke may be highly variable, even within the same community.

In the univariate analysis, although several demographic features were associated with HRQoL, only age emerged as an independent predictor of HRQoL. Although in this study and others age was a determinant of HRQoL, in some studies, age was not found to be a predictor of HRQoL. ${ }^{30-33}$ This shows that the association between HRQoL and age is not conclusive and that further studies are required to elucidate the actual effects of age on HRQoL. This is also the situation of sex, which was not a determinant of HRQoL in this study but has otherwise been reported by several studies as a determinant of HRQoL. ${ }^{31,32,34,35} \mathrm{HRQoL}$ is known to be affected by culture differences, ${ }^{2,4,32}$ and this may partly explain the variations in HRQoL and demographic factors.

Several clinical parameters were determinants of $\mathrm{HRQoL}$ in this study. Stroke severity was a very important determinant of HRQoL among the stroke survivors, as it affected almost all the HRQoL domains. This observation concurs with other studies that have consistently reported mild stroke as a determinant of HRQoL. ${ }^{17,32,35-37}$ In light of this, it is encouraging that the majority of the stroke survivors in the current study (52.6\%) had experienced mild stroke. The association of better psychoemotional HRQoL with longer stroke duration may be a result of response shift and coping strategies, which are developed over time. ${ }^{15,38}$ However, the reverse of this was observed for the spirit domain, as longer stroke duration determined poorer HRQoL. This observation could be a result of unfulfillment of expected recovery from stroke by spiritual methods with time. Contrary to other studies, number of strokes affected HRQoL. ${ }^{31,32}$ The association of a higher frequency of stroke attacks with poorer cognitive HRQoL is consistent with the observation that this domain was relatively more susceptible to impairment in a stroke attack, as shown by its lowest HRQoL score (57.7 \pm 16.2$)$. This may be further related to the fact that the brain, which is the seat of cognitive functions, is also the organ affected by stroke..$^{39,40}$ The side of the body affected by stroke and stroke subtype did not show any relationship with HRQoL, an observation that agrees with other studies. ${ }^{32-35}$ This implies that the evolution of stroke subtypes in Ghana, as described previously, has little relevance for HRQoL. In line with a Nigerian study, ${ }^{32}$ negative emotions determined poor psychoemotional HRQoL, whereas higher frequency of laughter determined better HRQoL for a wide range of domains, making the latter an important determinant of HRQoL. Stroke risk factors appear to have little relationship with HRQoL, as none of them emerged as independent determinants of HRQoL in this study.

We conclude that the population of Ghanaian stroke survivors investigated has a mean age of less than 60 years and that about one-half of them have mild stroke. Ischemic stroke is the predominant stroke subtype among the stroke survivors, whereas hypertension is the predominant stroke risk factor. The most impaired HRQoL domains of the stroke survivors are physical, psychoemotional, and cognitive domains. Determinants of HRQoL of the stroke survivors cover a wide spectrum of demographic, clinical, and psychological features, some of which are modifiable and can therefore be modulated to achieve improved HRQoL.

There are a few limitations of the study. Although the stroke survivors and controls were age- and sex-matched, several factors including education, income level, and marital status were significantly different between the two groups. This could have affected the HRQoL comparisons between the stroke survivors and controls. In addition, unlike the stroke survivors, we did not investigate comorbidities among the control group. Another limitation of the study is that we assessed HRQoL of a few stroke survivors on the basis of responses provided by their caregivers, as these stroke patients were unable to communicate. The information provided by a caregiver may not be a completely true representation of the subjective reflection of life experiences of the stroke patient.

\section{Acknowledgments}

The study was funded through a grant from the University of Iceland, and the funding received is gratefully acknowledged. The authors thank all the study participants, especially the stroke patients. We also acknowledge the support offered by various physiotherapists during the data collection process. 


\section{Disclosure}

The authors report no conflicts of interest in this work.

\section{References}

1. Salter KL, Moses MB, Foley NC, Teasell RW. Health-related quality of life after stroke: what are we measuring? Int J Rehabil Res. 2008;31(2):111-117.

2. Owolabi MO, Ogunniyi A. Profile of health-related quality of life in Nigerian stroke survivors. Eur J Neurol. 2009;16(1):54-62.

3. Hopman WM, Verner J. Quality of life during and after inpatient stroke rehabilitation. Stroke. 2003;34(3):801-805.

4. Hackett ML, Duncan JR, Anderson CS, Broad JB, Bonita R. Healthrelated quality of life among long-term survivors of stroke: results from the Auckland Stroke Study, 1991-1992. Stroke. 2000;31(2):440-447.

5. Bravata DM, Wells CK, Gulanski B, et al. Racial disparities in stroke risk factors: the impact of socioeconomic status. Stroke. 2005;36(7):1507-1511.

6. Connor MD, Walker R, Modi G, Warlow CP. Burden of stroke in black populations in sub-Saharan Africa. Lancet Neurol. 2007;6(3):269-278.

7. de-Graft Aikins A. Ghana's neglected chronic disease epidemic: a developmental challenge. Ghana Med J. 2007;41(4):154-159.

8. Acheampong JA. Stroke: Risk factors and factors predicting survival. Ghana Med J. 2001;35(4):148-149.

9. Wiredu EK, Nyame PK. Stroke mortality in Accra: A study of risk factors. Ghana Med J. 2001;35(4):151-161.

10. Anim JT, Kofi AD. Hypertension, cerebral vascular changes and stroke in Ghana. 1. Microaneurysm formation and stroke. J Pathol. 1984;143(3):177-182.

11. Agyemang C, Attah-Adjepong G, Owusu-Dabo E, et al. Stroke in Ashanti region of Ghana. Ghana Med J. 2012;46(2):12-17.

12. Ojo Owolabi M. HRQoLISP-26: A Concise, Multiculturally Valid, Multidimensional, Flexible, and Reliable Stroke-Specific Measure. ISRN Neurol. 2011;2011:295096.

13. Owolabi MO, Platz T. Proposing the Stroke Levity Scale: a valid, reliable, simple, and time-saving measure of stroke severity. Eur J Neurol. 2008;15(6):627-633.

14. Owolabi MO, Ugoya S, Platz T. Racial disparity in stroke risk factors: the Berlin-Ibadan experience; a retrospective study. Acta Neurol Scand. 2009;119(2):81-87.

15. King RB. Quality of life after stroke. Stroke. 1996;27(9):1467-1472.

16. Paul SL, Sturm JW, Dewey HM, Donnan GA, Macdonell RA, Thrift AG. Long-term outcome in the North East Melbourne Stroke Incidence Study: predictors of quality of life at 5 years after stroke. Stroke. 2005;36(10):2082-2086.

17. Jaracz K, Kozubski W. Quality of life in stroke patients. Acta Neurol Scand. 2003;107(5):324-329.

18. Edington GM. Cardiovascular disease as a cause of death in the Gold Coast African. Trans R Soc Trop Med Hyg. 1954;48(5):419-425.

19. Yates PO. A change in the pattern of cerebrovascular disease. Lancet. 1964;1(7324):65-69.

20. O'Donnell MJ, Xavier D, Liu L, et al; INTERSTROKE investigators. Risk factors for ischaemic and intracerebral haemorrhagic stroke in 22 countries (the INTERSTROKE study): a case-control study. Lancet. 2010;376(9735):112-123.
21. Bwala SA. Stroke in a subsaharan Nigerian hospital - a retrospective study. Trop Doct. 1989;19(1):11-14.

22. Hajat C, Dundas R, Stewart JA, et al. Cerebrovascular risk factors and stroke subtypes: differences between ethnic groups. Stroke. 2001;32(1):37-42.

23. Amoah AG. Hypertension in Ghana: a cross-sectional community prevalence study in greater Accra. Ethn Dis. 2003;13(3):310-315.

24. Bosu WK. Epidemic of hypertension in Ghana: a systematic review. BMC Public Health. 2010;10:418.

25. Kim HC, Nam CM, Jee SH, Suh I. Comparison of blood pressureassociated risk of intracerebral hemorrhage and subarachnoid hemorrhage: Korea Medical Insurance Corporation study. Hypertension. 2005;46(2):393-397.

26. Ebrahim S, Sung J, Song YM, Ferrer RL, Lawlor DA, Davey Smith G. Serum cholesterol, haemorrhagic stroke, ischaemic stroke, and myocardial infarction: Korean national health system prospective cohort study. BMJ. 2006;333(7557):22.

27. Owolabi MO, Agunloye AM. Risk factors for stroke among patients with hypertension: a case-control study. J Neurol Sci. 2013; 325(1-2):51-56.

28. Owolabi MO, Agunloye AM. Which risk factors are more associated with ischemic rather than hemorrhagic stroke in black Africans? Clin Neurol Neurosurg. 2013;115(10):2069-2074.

29. Clarke P, Marshall V, Black SE, Colantonio A. Well-being after stroke in Canadian seniors: findings from the Canadian Study of Health and Aging. Stroke. 2002;33(4):1016-1021.

30. Lai SM, Studenski S, Duncan PW, Perera S. Persisting consequences of stroke measured by the Stroke Impact Scale. Stroke. 2002;33(7):1840-1844.

31. Gokkaya NK, Aras MD, Cakci A. Health-related quality of life of Turkish stroke survivors. Int J Rehabil Res. 2005;28(3):229-235.

32. Owolabi MO. Determinants of health-related quality of life in Nigerian stroke survivors. Trans R Soc Trop Med Hyg. 2008;102(12): 1219-1225.

33. Gurcay E, Bal A, Cakci A. Health-related quality of life in first-ever stroke patients. Ann Saudi Med. 2009;29(1):36-40.

34. Wyller TB, Holmen J, Laake P, Laake K. Correlates of subjective well-being in stroke patients. Stroke. 1998;29(2):363-367.

35. Kong KH, Yang SY. Health-related quality of life among chronic stroke survivors attending a rehabilitation clinic. Singapore Med J. 2006;47(3):213-218.

36. Haacke C, Althaus A, Spottke A, Siebert U, Back T, Dodel R. Longterm outcome after stroke: evaluating health-related quality of life using utility measurements. Stroke. 2006;37(1):193-198.

37. Müller-Nordhorn J, Nolte CH, Rossnagel K, et al. The use of the 12-item short-form health status instrument in a longitudinal study of patients with stroke and transient ischaemic attack. Neuroepidemiology. 2005;24(4):196-202.

38. Addington-Hall J, Kalra L. Who should measure quality of life? BMJ. 2001;322(7299):1417-1420.

39. Robinson RG. The Clinical Neuropsychiatry of Stroke: Cognitive, Behavioural and Emotional Disorders Following Vascular Brain Injury. New York: Cambridge University Press; 2006.

40. Gurenlian JR, Kleiman C. Cerebrovascular accident. Access. 2002; $6(16): 40-47$.
Clinical Interventions in Aging

\section{Publish your work in this journal}

Clinical Interventions in Aging is an international, peer-reviewed journal focusing on evidence-based reports on the value or lack thereof of treatments intended to prevent or delay the onset of maladaptive correlates of aging in human beings. This journal is indexed on PubMed Central, MedLine,

\section{Dovepress}

CAS, Scopus and the Elsevier Bibliographic databases. The manuscript management system is completely online and includes a very quick and fair peer-review system, which is all easy to use. Visit http://www.dovepress. com/testimonials.php to read real quotes from published authors. 